\title{
Una propuesta para aprender ciencias y matemáticas mediante el juego: Construimos nuestra ciudad
}

\author{
María Velázquez Pérez, Alicia Fernández Oliveras y María Luisa Oliveras. Universidad de \\ Granada \\ Recepción: 12 de abril de 2015 | Revisión: 30 de mayo de 2015 | Aceptación/Publicación: 24 de julio de 2015 \\ Correspondencia: vpmaria@correo.ugr.es | alilia@ugr.es | oliveras@ugr.es | http://hdl.handle.net/10481/37122
}

Resumen: Proponemos un juego educativo para abordar de forma globalizada contenidos científicos y matemáticos propios de la etapa de Educación Infantil. Se decidió realizar un juego de mesa situado en un contexto urbano con el que los niños pudieran identificarse fácilmente y comprobar que las ciencias y las matemáticas están también presentes en su ciudad. Los aspectos más representativos del juego creado se resumen en dos fichas: una de carácter técnico y otra, didáctica.

Palabras clave: Didáctica de las Ciencias Experimentales | Didáctica de la Matemática | Formación del Profesorado | Investigación-Acción | Juegos Educativos

\section{A PROPOSAL FOR SCIENCE AND MATHEMATICS LEARNING THROUGH PLAY: BUILDING OUR CITY}

Abstract: We propose an educational game to address, in a "globalized" way, scientific and mathematical content specific to kindergarten education. We decided to make a board game set in an urban context with which children could easily identify themselves, and discover that science and mathematics are also present in their city. The most representative aspects of the game created are summarized on two index cards: one technical and the other didactic.

Keywords: Science Education | Mathematical Education | Teacher Training | Action Research | Educational Games

Agradecimientos: Al Secretariado de Innovación Docente del Vicerrectorado de Ordenación Académica y Profesorado de la Universidad de Granada (España), por conceder el Proyecto de Innovación Docente 2013-2016.

\section{Introducción}

Según la Real Academia Española (s.f.), juego significa: "1). m. Acción y efecto de jugar. 2). m. Ejercicio recreativo sometido a reglas, y en el cual se gana o se pierde."

Pero el significado de juego va mucho más allá, no solo se rige por la acción de jugar en la que solo se puede ganar o perder, sino que tiene multitud de implicaciones educativas que permiten el desarrollo del niño. Investigaciones realizadas señalan que los docentes en formación de Educación Infantil encuentran claras relaciones entre el juego y el desarrollo del pensamiento científico y matemático (Fernández-Oliveras y Oliveras, 2014b, 2015).

Por ello, nos propusimos diseñar y realizar un juego que permitiese abordar la integración de las ciencias y las matemáticas en las aulas de infantil, con un enfoque lúdico y globalizador. Decidimos situar el juego en contexto urbano, con el que los niños pudieran identificarse fácilmente, comprobando así, que las ciencias y las matemáticas están también presentes en su ciudad.

Algunos de los objetivos que se pretenden conseguir a través del juego, dados en la ORDEN ECI/3960/2007, de 19 de diciembre, figuran en la Tabla 1. 
Tabla 1. Objetivos del juego educacional presentes en la ORDEN ECI/3960/2007.

\begin{tabular}{|c|c|}
\hline Áreas & Objetivos \\
\hline \multirow[t]{2}{*}{$\begin{array}{l}\text { Área 1. Conocimiento de sí mismo } \\
\text { y autonomía personal }\end{array}$} & $\begin{array}{l}\text { 5. Realizar, de manera cada vez más autónoma, } \\
\text { actividades habituales y tareas sencillas para } \\
\text { resolver problemas de la vida cotidiana, } \\
\text { aumentando el sentimiento de autoconfianza y la } \\
\text { capacidad de iniciativa. }\end{array}$ \\
\hline & $\begin{array}{l}\text { 7. Adecuar su comportamiento a las necesidades } \\
\text { y requerimientos de los otros, desarrollando } \\
\text { actitudes y hábitos de respeto, ayuda y } \\
\text { colaboración, evitando actitudes de sumisión o } \\
\text { dominio. }\end{array}$ \\
\hline \multirow[t]{3}{*}{ Área 2. Conocimiento del entorno } & $\begin{array}{l}\text { 1. Observar y explorar de forma activa su entorno } \\
\text { físico, natural y social, desarrollar el sentido de } \\
\text { pertenencia al mismo, mostrando interés por su } \\
\text { conocimiento, y desenvolverse en él con cierta } \\
\text { seguridad y autonomía. }\end{array}$ \\
\hline & $\begin{array}{l}\text { 5. Representar atributos de elementos y } \\
\text { colecciones, y establecer relaciones de } \\
\text { agrupamientos, clasificación, orden y } \\
\text { cuantificación, iniciándose en las habilidades } \\
\text { matemáticas. }\end{array}$ \\
\hline & $\begin{array}{l}\text { 6. Interesarse por el medio natural, observar y } \\
\text { reconocer animales, plantas, elementos y } \\
\text { fenómenos de la naturaleza, experimentar, hablar } \\
\text { sobre ellos y desarrollar actitudes de curiosidad. }\end{array}$ \\
\hline \multirow{3}{*}{$\begin{array}{l}\text { Área } 3 \text {. Lenguajes: comunicación } \\
\text { y representación }\end{array}$} & $\begin{array}{l}\text { 5. Acercarse a las producciones de tradición } \\
\text { cultural. Comprender, recitar, contar y recrear } \\
\text { algunos textos literarios mostrando actitudes de } \\
\text { valoración, disfrute e interés hacia ellos. }\end{array}$ \\
\hline & $\begin{array}{l}\text { 6. Desarrollar la curiosidad y la creatividad } \\
\text { interactuando con producciones plásticas, } \\
\text { audiovisuales y tecnológicas, teatrales, musicales, } \\
\text { o danzas, mediante el empleo de técnicas } \\
\text { diversas. }\end{array}$ \\
\hline & $\begin{array}{l}\text { 7. Iniciarse en los usos sociales de la lectura y la } \\
\text { escritura explorando su funcionamiento y } \\
\text { valorándolas como instrumento de comunicación, } \\
\text { información y disfrute. }\end{array}$ \\
\hline
\end{tabular}

\section{DISEÑO DEL JUEGO}

Se decidió realizar un juego de mesa que se desarrollara en un ambiente donde la protagonista fuera una ciudad cualquiera y sus edificios más característicos. En el tablero se representaría la ciudad sin esos edificios, que los jugadores tendrían que ir consiguiendo tras superar distintas pruebas con contenidos científicos y matemáticos, contextualizadas en cada edificio de la ciudad. Se trataría de un juego colaborativo en el que todos los participantes forman parte de un mismo equipo.

En la Figura 1 aparecen los primeros bocetos del tablero y algunos de los bocetos para las pruebas que incluye el juego. 

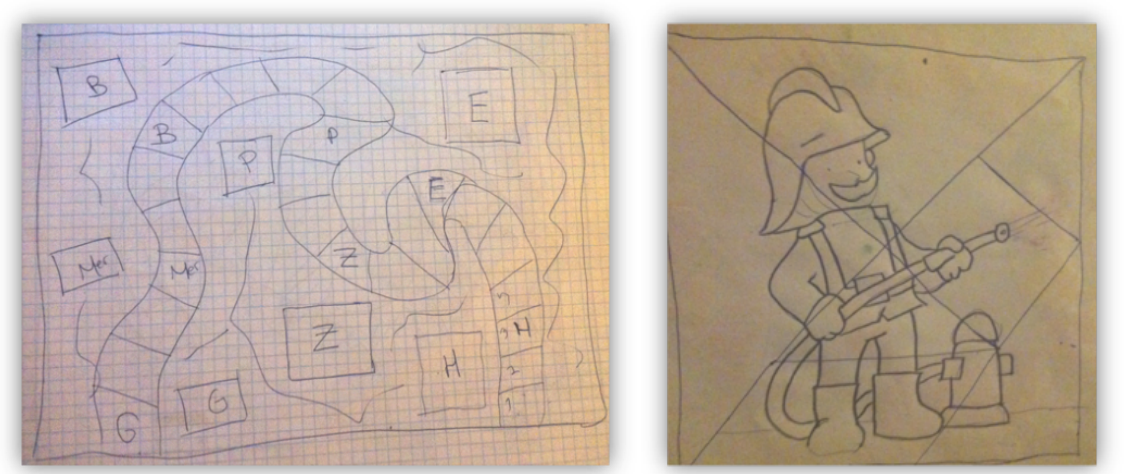

Figura 1: Bocetos del tablero y de algunas de las pruebas que incluye el juego.

\section{ELABORACIÓN}

Seguidamente se describen brevemente los distintos elementos componentes del juego y su preparación:

- Tablero: En una cartulina blanca se ha dibujo una ciudad, en la que no están presentes ninguno de los edificios que la componen, solo la carretera por la que los jugadores irán avanzando. A lo largo de la carretera encontraran pasos de peatones en los que los jugadores deberán pararse para superar unas pruebas y conseguir los edificios para así completar la ciudad.

- Edificios: Se han dibujado en una cartulina blanca, se han recortado y se le han pegado velcro por detrás. Son siete: hospital, escuela, estación de bomberos, policía, mecánico, zoológico y mercado.

- Adivinanzas: se han escrito siete adivinanzas diferentes en cartulinas de color naranja y rosa, cada una relacionada con uno de los edificios.

- Tarjetas de las pruebas: son catorce tarjetas, están realizadas con cartulina de color rojo y verde y en ellas se indican los elementos para realizar las pruebas.

- Pruebas de los diferentes edificios: se han realizado catorce pruebas, dos diferentes para cada edificio, una de matemáticas y otra de ciencias. Estas pruebas han sido totalmente de elaboración propia.

- Medallas: Las medallas están creadas con goma-eva, cartulina y lana, donde se especifica la propiedad de la que serán dueños.

- Ruleta: para la creación de la ruleta se ha utilizado una tapadera. Encima de ella se ha pegado una cartulina de color rojo y verde y se ha hecho un agujero en el centro de la misma. En este agujero se ha colocado una brida enganchada a un tornillo y a una arandela para que pueda girar fácilmente

- Coche de juguete: es la pieza que los jugadores utilizaran para desplazarse por el tablero.

\section{FICHAS TÉCNICA Y DIDÁCTICA DEL JUEGO}

La información más relevante sobre el juego creado se recoge en las Tablas 2 y 3 , cuya estructura procede de los materiales facilitados en una asignatura del Grado en Maestro de Educación Infantil de la Universidad de Granada (Fernández-Oliveras y Oliveras, 2014a). 
Tabla 2. Ficha técnica del juego "Construimos la ciudad".

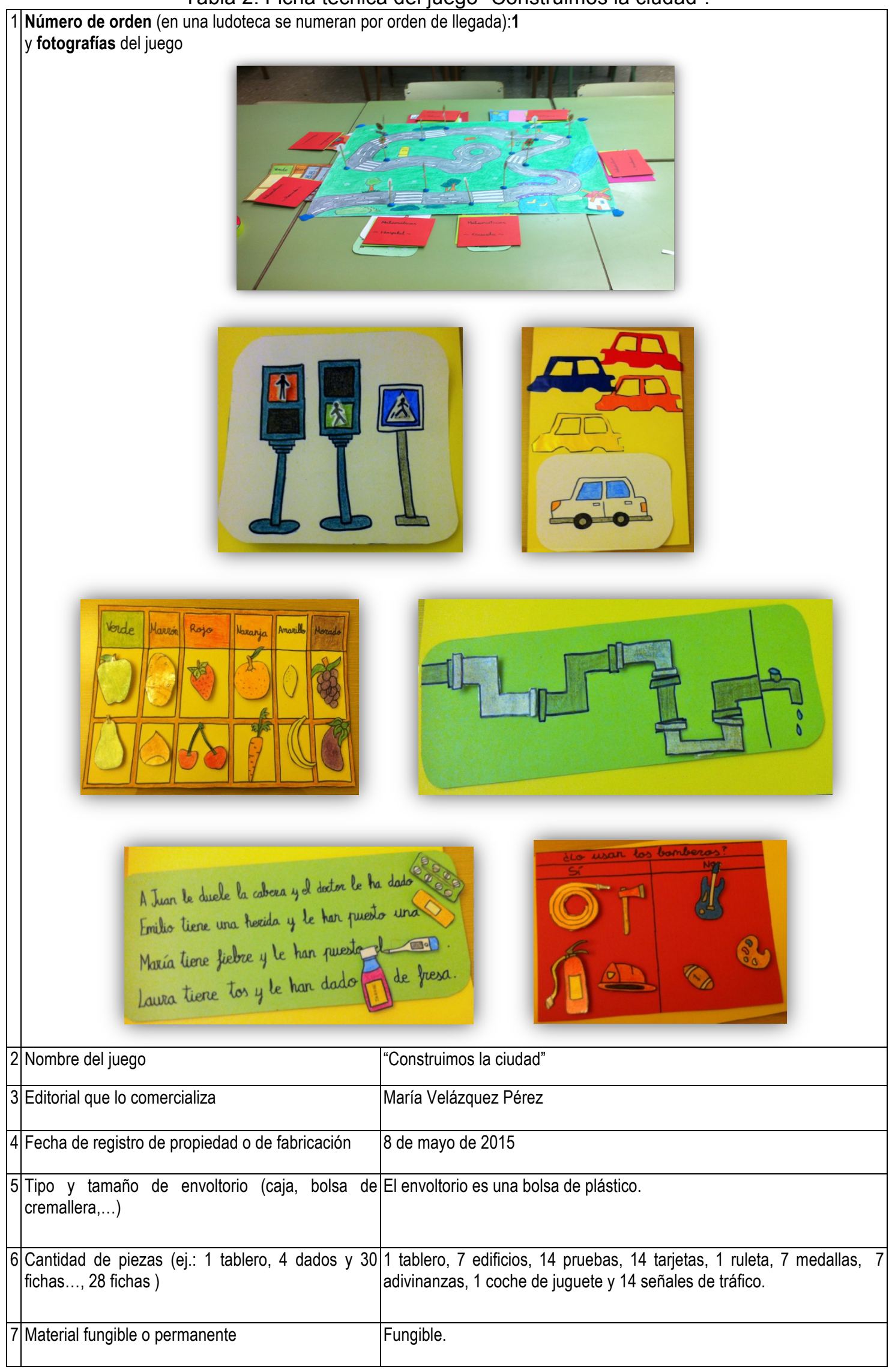


Tabla 3. Ficha didáctica del juego "Construimos la ciudad".

\begin{tabular}{|c|c|}
\hline Nombre del juego & “Construimos la ciudad" \\
\hline Piezas y material: descripción & $\begin{array}{l}\text { La mayoría de las piezas están hechas con cartulina, ceras de colores y rotuladores, a } \\
\text { pesar de otros materiales no tan predominantes como la gomaeva, lana y palillos. }\end{array}$ \\
\hline Lugar de fabricación & España \\
\hline Homologación [Sí, en qué país(es): /No] & No \\
\hline $\begin{array}{l}\text { Cualidades intrínsecas de las piezas: } \\
\text { formas y color, material del que están } \\
\text { hechas }\end{array}$ & $\begin{array}{l}\text {-Tablero de colores de forma rectangular formado por: cartulina, ceras de colores, } \\
\text { rotulador y velcro. } \\
\text {-Edificios de colores con forma cuadrada y fabricados con: cartulina, ceras de colores y } \\
\text { rotulador. } \\
\text {-Señales redondas de color verde y rojo, realizados con: cartulina, palillos, ceras de } \\
\text { colores y rotuladores. } \\
\text {-Pruebas rectangulares de colores muy variados, realizadas con: cartulina, ceras de } \\
\text { colores, rotuladores, velcro y papel charol. } \\
\text {-Tarjetas rojas y verdes de tamaño rectangular y fabricado con: cartulina. } \\
\text {-Ruleta redonda de color rojo y verde, construida con: tapadera redonda, cartulina, ceras } \\
\text { de colores, arandela, tornillo y brida. } \\
\text {-Medallas redondas de colores fabricadas con: gomaeva, cartulina y lana. } \\
\text {-Coche pequeño de juguete. }\end{array}$ \\
\hline $\begin{array}{l}\text { Cualidades relativas de las piezas: } \\
\text { tamaños, calor, texturas, volumen }\end{array}$ & La mayoría de las piezas tienen un tamaño grande, de textura suave y sin volumen. \\
\hline Dinámica del juego y reglas: explicación & $\begin{array}{l}\text { El juego consiste en construir una ciudad. Para ello, se formará un único equipo de niños } \\
\text { y juntos deberán cooperar para conseguir los edificios que le faltan a la ciudad. } \\
\text { Deberán desplazarse a través de la carretera de la ciudad con un coche de juguete y y } \\
\text { cada vez que lleguen a un paso de peatones en el que hay colocada una señal, deberán } \\
\text { pararse. En esta señal se indica que deben girar una ruleta. Si la ruleta que han girado } \\
\text { marca el color verde, deberán realizar unas pruebas de ciencias y si marca el color rojo, } \\
\text { serán de matemáticas. Conforme vayan superando las pruebas, irán consiguiendo los } \\
\text { edificios. A su vez, tras la consecución de un edificio, se recitará una adivinanza y quien } \\
\text { la acierte conseguirá una medalla en la que se indica que es el director de ese edificio } \\
\text { que han conseguido. } \\
\text { El objetivo del juego es que entre todos se ayuden para conseguir construir la ciudad. }\end{array}$ \\
\hline $\begin{array}{l}\text { Peligrosidad/riesgos (en las piezas o en } \\
\text { su uso con determinadas reglas) }\end{array}$ & Riesgo de ingerir las piezas más pequeñas. \\
\hline Tiempo de preparación & Medio. \\
\hline Duración [corta/media/larga] & Media. \\
\hline $\mathrm{N}^{0}$ de jugadores & De 2 a 4 jugadores pero formando siempre un único equipo. \\
\hline Edad preferente de los jugadores & 5 años \\
\hline De uso en interior/exterior & Interior. \\
\hline $\begin{array}{l}\text { Tipos de acciones de los jugadores } \\
\text { primordialmente (físicas, mentales, } \\
\text { ambas) }\end{array}$ & Acciones mentales. \\
\hline Sentidos que se utilizan & Vista y tacto. \\
\hline $\begin{array}{l}\text { Habilidades/capacidades } 0 \text { destrezas } \\
\text { que favorece (psicomotrices, } \\
\text { cognitivas...) }\end{array}$ & Favorece las habilidades cognitivas. \\
\hline Objetivos propios del juego & $\begin{array}{l}\text {-Propiciar la participación activa de los jugadores. } \\
\text {-Fomentar el trabajo en equipo y la socialización entre ellos. } \\
\text {-Desarrollar el razonamiento. } \\
\text {-Desarrollar la observación. } \\
\text {-Desarrollar la concentración. } \\
\text {-Reforzar conocimientos previos. } \\
\text {-Trabajar aspectos matemáticos propios del entorno urbano. } \\
\text {-Trabajar aspectos científicos propios del entorno urbano. } \\
\text {-Estimular la imaginación. } \\
\text {-Desarrollar la comunicación y el lenguaje. }\end{array}$ \\
\hline Historia y cultura origen del juego & $\begin{array}{l}\text { Se basa en las alfombras que usaban los niños pequeños en forma de carretera y en el } \\
\text { conocido juego Monopoly. }\end{array}$ \\
\hline Coste económico (estimado o conocido) & 5 euros. \\
\hline Contenidos matemáticos & Tamaños, números, formas geométricas, asociaciones, conteo, clasificación. \\
\hline Contenidos científicos & $\begin{array}{l}\text { Colores y mezclas de colores, animales, frutas, medicina, señales de tráfico, } \\
\text { herramientas y utensilios (para la labor de un bombero). }\end{array}$ \\
\hline tocorrección [Sí, de qué & No. \\
\hline
\end{tabular}




\section{Reflexión final}

En este artículo se ha resumido el diseño y elaboración de un juego educativo adaptado a la etapa de infantil, haciendo especial énfasis en un enfoque globalizador de la enseñanza de las ciencias y las matemáticas.

\section{Referencias}

Fernández-Oliveras, A. y Oliveras, M. L. (2014a). Playing for science and mathematics education: an experience for pre-service kindergarten teacher training. En Costa M. F. M., Pombo P., Dorrío B.V. (Eds.), Hands-on Science. Science Education with and for Society (180 -183). Braga: Hands-on Science Network.

Fernández-Oliveras, A. y Oliveras, M. L. (2014b). Pre-service kindergarten teachers' conceptions of play, science, mathematics, and education. Procedia - Social and Behavioral Sciences, 152, 856-861. DOI:10.1016/j.sbspro.2014.09.334.

Fernández-Oliveras, A. y Oliveras, M. L. (2015). Conceptions of science, mathematics, and education of prospective kindergarten teachers in a play-based training. International Journal on Advances in Education Research, 2(1), 37-48.

Orden, E. C. I. (2008). 3960/2007, de 19 de diciembre, por la que se establece el currículo y se regula la ordenación de la educación infantil. Boletín Oficial del Estado, 5, 1016-36.

Real Academia Española. (s. f.). Juego [artículo enmendado]. En Diccionario de la lengua española (avance de la 23.a ed.). Recuperado de http://lema.rae.es/drae/srv/search?id=W8r2F0KpP2x5DoSLxvf 